\title{
La formation en gestion et le développement durable
}

\author{
Denis Bourque \\ Université du Québec à Chicoutimi
}

Le législateur québécois a adopté, le 13 avril 2006, la Loi sur le développement durable. J'aborderai brièvement les objectifs de cette loi, ses champs d'application, les mesures prévues pour assurer sa mise en œuvre d'une manière efficace et, enfin, les répercussions de l'adoption de cette loi sur ce qui est convenu d'appeler «la formation en gestion» ${ }^{1}$.

\section{1- Les objectifs de la Loi sur le développement durable}

D'entrée de jeu, le législateur québécois précise que la loi sur le développement durable a pour objet d'instaurer un «nouveau cadre de gestion au sein de l'administration» afin que l'exercice des
Le législateur québécois affirme qu'il adopte un concept de gouvernance fondé sur le développement durable ${ }^{2}$ les mesures qui y sont prévues visent plus particulièrement à réaliser le virage nécessaire au sein de la société face aux modes de développement non viable, en intégrant davantage la recherche pouvoirs et des responsabilités dévolus à cette administration s'inscrive dans la recherche d'un développement durable. Cette législation prévoit que le développement durable « s'entend d'un développement qui répond aux besoins du présent sans compromettre la capacité des générations futures à répondre aux leurs ». Selon le texte législatif, le développement durable s'appuie sur une vision à long terme qui prend en compte le caractère indissociable des dimensions environnementale, sociale et économique des activités de développement. La loi ajoute que d'un développement durable à tous les niveaux et dans toutes les sphères d'intervention de l'Administration, c'est-à-dire dans ses politiques, ses programmes et ses actions particularisées. Les mesures législatives précisent qu'elles visent également à assurer la cohérence des actions gouvernementales en matière de développement durable ainsi qu'à favoriser l'imputabilité de l'Administration en cette matière. D'une façon claire, le législateur québécois affirme qu'il adopte un concept de gouvernance fondé sur le développement durable $^{2}$.

\section{2- Le champ d'application de la Loi sur le développement durable}

La Loi sur le développement durable s'applique d'abord au gouvernement, au Conseil exécutif, au Conseil du Trésor, aux ministères, de même qu'aux organismes et aux entreprises du gouvernement visés par la Loi sur le vérificateur général. Cependant, le gouvernement peut déterminer, par décret, à quelle date et selon quelles conditions cette loi peut s'appliquer aux universités, aux municipalités et aux hôpitaux ${ }^{3}$. 


\section{3- La mise en œuvre du développement durable}

La mise en œuvre du développement durable au sein de l'Administration s'appuie sur les principes faisant partie d'une stratégie de développement durable, laquelle est adoptée par le gouvernement; elle se réalise également dans le respect des principes prévus dans la loi elle-même. Voici d'ailleurs deux des principes inclus dans la loi :

a) «internalisation des coûts»: c'est-à-dire que la valeur des biens et des services doit refléter l'ensemble des coûts qu'ils occasionnent à la société durant tout leur cycle de vie, de leur conception jusqu'à leur consommation et leur disposition finale ${ }^{4}$.

b) «pollueur payeur»: les personnes qui génèrent de la pollution ou dont les actions dégradent autrement l'environnement doivent assumer leur part des coûts des mesures de prévention, de réduction et de contrôle des atteintes à la qualité de l'environnement ${ }^{5}$.

Reste à voir comment les sciences comptables et les sciences administratives vont actualiser ces principes «d'internalisation des coûts» et de «pollueur payeur» compte tenu de la vive concurrence entre les entreprises ${ }^{6}$.

Quant à la stratégie de développement durable que doit adopter le gouvernement, la loi prévoit que celle-ci doit exposer la vision retenue, les enjeux, les orientations ou les axes d'intervention, ainsi que les objectifs que doit poursuivre l'Administration en matière de développement durable ${ }^{7}$.

La loi prévoit également que la stratégie doit aborder le développement d'outils ou de grilles d'aide à la conception, à la décision et à l'analyse de projets en regard du développement durable, entre autres pour prendre en compte l'ensemble des principes ou pour mettre en application des approches liées à ceux-ci, notamment quant au cycle de vie des produits et des services ${ }^{8}$.

Afin de centrer ses priorités et de planifier ses actions de manière à tendre vers un développement durable en conformité avec la stratégie du gouvernement, chaque ministère, organisme et entreprise compris dans l'Administration identifie dans un document qu'il doit rendre publique les objectifs particuliers qu'il entend poursuivre pour contribuer à la mise en œuvre progressive de la stratégie, ainsi que les activités ou les interventions qu'il prévoit réaliser à cette fin, directement ou en collaboration avec un ou plusieurs intervenants de la société 9 .

En matière de reddition de comptes et d'imputabilité, chaque ministère, organisme et entreprise compris dans l'Administration fait état dans le rapport annuel de ses activités :

a) Des objectifs particuliers qu'il s'était fixés, en conformité avec ceux de la stratégie, pour contribuer au développement durable et à la mise en œuvre progressive de la stratégie ou, le cas échéant, des motifs pour lesquels aucun objectif particulier n'a été identifié pour l'année vu le contenu de la stratégie adoptée;

b) Des différentes activités ou interventions qu'il a pu ou non réaliser durant l'année en vue d'atteindre les objectifs identifiés, ainsi que du degré d'atteinte des résultats qu'il s'était fixés, en précisant les indicateurs de développement durable ainsi que les autres moyens ou mécanismes de suivi retenus ${ }^{10}$. 
La loi crée également un poste de commissaire du développement durable qui, sous l'autorité du vérificateur général, présente chaque année un rapport à l'Assemblée nationale dans lequel il peut formuler ses recommandations ayant trait à l'application de la Loi sur le développement durable et ses commentaires concernant les principes, les procédures ou les autres moyens employés en matière de développement durable par l'Administration. Il peut porter à l'attention de l'Assemblée nationale tout sujet ou tout cas qui découle de ses travaux de vérification ou d'enquête en matière de développement durable ${ }^{11}$.

Enfin, la Loi sur le développement durable modifie la Charte des droits et libertés de la personne ${ }^{12}$ par l'insertion, après l'article $46 \mathrm{de}$ la Charte, du suivant :

« 46.1 Toute personne a droit, dans la mesure et suivant les normes prévues par la loi, de vivre dans un environnement sain et respectueux de la biodiversité. $»^{13}$

\section{4- Les conséquences de l'adoption de cette loi sur la formation en gestion.}

Relativement aux conséquences de l'adoption de la Loi sur le développement durable sur la formation en gestion, la première est certainement celle d'éduquer et de conscientiser les étudiants sur le sens et la portée du langage utilisé dans la loi elle-même et saisir le sens et la portée des gestes et des actions qui en découlent.

En termes clairs, tout cet univers que l'on appelle « gestion de projet », « analyse de projet » et « management de projet » est actuellement soumis à un véritable tsunami d'origine législative. Situons bien le contexte. Les organismes gouvernementaux et paragouvernementaux dont les ministères et les entreprises du gouvernement (et bientôt les municipalités, les hôpitaux et les universités) accordent chaque année des contrats de plusieurs milliards de dollars aux entreprises privées. Encore faut-il que les gestionnaires des entreprises privées saisissent les nuances $\mathrm{du}$ nouveau langage utilisé par l'Administration publique, notamment en ce qui a trait au développement et à l'utilisation d'indicateurs, d'outils ou de grilles d'aide à la conception, à la décision et à l'analyse de projets en regard du développement durable. Par exemple, comment les entreprises vontelles composer avec les nouveaux plans d'action des ministères et avec la liste des indicateurs de développement durable adoptés par le gouvernement? Comment ces mêmes entreprises vont-elles composer avec les principes «d'internalisation des coûts» et de «pollueur-payeur»? ${ }^{14}$

La semaine dernière, l'activité économique québécoise et canadienne a été marquée par le début du «marché climatique» de la Bourse de Montréal qui a lancé ses nouveaux contrats à terme MCX de crédits d'émission de GES carbone. La bourse du carbone existe maintenant au Canada. Cette bourse du carbone fonctionne de quelle manière? Quels objectifs poursuit-elle?

La semaine prochaine, le ministre québécois des ressources naturelles déposera un projet de loi qui établira un nouveau régime forestier qui, semble-t-il, se raccrochera au concept de développement durable et qui créera une «bourse de la forêt». Cette bourse de la forêt fonctionnera de quelle manière? Quels objectifs poursuivra-t-elle?

L'Université du Québec à Chicoutimi fait des efforts afin de relever ce défi éducatif. D'abord, dans son schéma directeur 20062011, adopté en décembre 2006, notre université affirme qu'elle veut jouer pleinement son rôle comme acteur de 
développement durable. Ensuite dans plusieurs de nos programmes de deuxième cycle, dont le programme court de deuxième cycle en écoconseil et le diplôme d'études supérieures spécialisés en éco-conseil, nous abordons le contenu de la Loi sur le développement durable et la problématique relative à la gestion des gaz à effet de serre.

Des entreprises privées comme Bell Canada, Bombardier, Desjardins participent également à l'effort éducatif. Notons que c'est une compagnie privée, Power Corporation, qui a invité, au début d'avril 2008, l'ancien viceprésident américain $\mathrm{Al}$ Gore pour sensibiliser la population aux questions reliées aux changements climatiques.

Cet effort éducatif en matière de développement durable constitue donc certainement l'un des volets du " défi de l'avenir » en matière de formation en gestion.

La deuxième conséquence de l'adoption de cette loi est celle d'éduquer et de conscientiser les étudiants sur l'existence d'une nouvelle réalité géopolitique qui rend plus complexe les relations d'affaires et qui appelle à la formation d'un esprit critique.

Le premier point qui caractérise cette nouvelle géopolitique c'est que plusieurs pays s'unissent pour essayer d'exercer une gouvernance basée sur le développement durable. Par exemple, l'union européenne, formée de 27 pays, s'attaque maintenant ouvertement au problème du dumping environnemental et, à plus court terme, aux importations en provenance des pays qui ne respectent pas les exigences du protocole de Kyoto. Le 24 avril 2008, l'Union européenne a adopté un rapport visant à jeter les bases d'une politique de développement durable encadrée par des instruments fondés sur le marché de l'internalisation des coûts environnementaux dans tous les biens et services et sur le principe du pollueur-payeur. L'Union européenne voudrait mettre en place une «taxe carbone» aux frontières de l'Europe pour tous les biens produits dans des pays étrangers qui ne sont pas liés au protocole de Kyoto ou qui n'en respectent pas les règles. Inutile de vous dire que cette initiative risque de rendre plus complexe les relations d'affaires avec l'Europe. L'Union européenne se doit de concilier la poursuite de ses efforts de réduction des émissions de $\mathrm{CO}_{2}$ avec les règles du commerce international, notamment celle de non-discrimination des partenaires commerciaux. À noter que l'Organisation mondiale du commerce (OMC) reconnaît maintenant les principes du développement durable et le droit de prendre des mesures pour protéger l'environnement et la santé publique. ${ }^{15}$

Cette initiative de l'Union européenne illustre l'importance du rôle de l'État dans la mise en œuvre du concept de développement durable.

Le 22 mai 2008, la banque mondiale rendait public le rapport de la Commission Croissance et Développement. La principale conclusion de ce rapport « est que la croissance indispensable pour faire reculer la pauvreté et assurer un développement durable réclame un État fort ». Les auteurs de ce rapport soulignent que «plus l'économie crôt, plus une administration publique active et pragmatique a un rôle crucial à jouer, ce qui implique une planification à long terme ». Ils attirent également notre attention sur le fait « qu'ignorer les problèmes environnementaux dans les premiers temps du développement ampute inévitablement la croissance à terme $\gg .{ }^{16}$

\section{La croissance indispensable pour faire reculer la pauvreté et assurer un \\ développement durable réclame un État fort}


Le deuxième point qui caractérise cette nouvelle réalité géopolitique c'est l'émergence de puissants acteurs non étatiques qui risquent de rendre très difficile l'exercice par les parlements de leurs pouvoirs législatifs afin de réaliser le virage nécessaire au sein de la société face aux modes de développement non viable. Par exemple, le bénéfice net de la compagnie Exxon en 2007 aurait placé cette pétrolière au $82^{\mathrm{e}}$ rang des pays au chapitre du PIB. Si maintenant on tenait compte du chiffre d'affaires, la compagnie Exxon avec ses 400 milliards de chiffre d'affaire, se situerait au $28^{\mathrm{e}}$ rang dans ce classement des pays par PIB. ${ }^{17}$

Le grand patron d'Occidental Petroleum a reçu une rémunération totale (salaire et levée d'options) de 415 millions en 2006, une marque qui n'a pas encore été égalée dans l'industrie du pétrole. Comment légiférer avec

\section{Conclusion}

La Loi sur le développement durable, avec ses dimensions tripolaires, est un appel à un nouveau « vivre ensemble ». L'adoption de cette loi et de sa stratégie de mise en œuvre constituent des avancées sociales importantes.

Les aspects de complexité auxquels j'ai fait allusion précédemment ajoutent à ceux déjà formulés par d'autres collègues qui ont abordé, entre autres, plus spécifiquement l'importante problématique de l'internormativité et de l'interdisciplinarité.

Le défi posé par la loi n'est pas marqué du coin de la confection de recettes magiques; il s'agit plutôt d'un défi marqué au coin de la des monstres comme Exxon et Occidental Petroleum.

La crise alimentaire qui sévit actuellement dans le monde est un autre point qui caractérise la nouvelle réalité géopolitique. Malgré cette crise alimentaire, le Canada adoptera probablement dans quelques jours un projet de loi permettant à l'industrie de l'éthanol de prendre son envol. Selon certains experts, cette décision fera grimper les prix des céréales, ce qui aggravera les problèmes de faim dans le monde. À noter que selon le bilan onusien GE04, depuis 1987, la population mondiale a augmenté de $34 \%$.

Cet effort éducatif en matière de géopolitique et cette conscientisation vis-à-vis de l'importance d'un État fort représente aussi l'un des volets du « défi de l'avenir » en matière de formation en gestion.

conscientisation à la complexité du « vivre ensemble ».

En clair, il s'agit d'initier l'étudiant à un monde beaucoup plus complexe tant en lui faisant comprendre qu'il y a une place pour sa créativité et que celle-ci d'ailleurs peut contribuer au bon fonctionnement de ce nouveau monde.

N'oublions pas que la première ligne de la loi parle de «gestion» et nos préoccupations ont trait à «la gestion». Le législateur québécois nous fait donc un clin d'œil. Essayons de relever le défi ensemble. 


\section{Notes et références}

1 Loi sur le développement durable, L.R.Q., C.D-8.1.1. Le projet de loi no. 118 (Loi sur le développement durable) a été sanctionné le 19 avril 2006 et est devenu le chapitre 3 des lois de 2006. Le Canada étant une fédération, le Parlement fédéral a, de son côté, adopté une loi relative au développement durable qui s'applique à ses champs de compétence.

2 Id., art. 1 et 2.

Id., art. 3 et 4.

Id., art. 6, par. p.

Id., art. 6, par. o.

6 Relativement au principe «pollueur payeur», notons que la Cour suprême du Canada, dans son jugement rendu le 20 novembre 2008 dans l'affaire Ciment du Saint-Laurent Inc., a reconnu l'existence d'une responsabilité sans faute reliée à l'application de l'article 976 du Code civil du Québec. La Cour souligne que cette responsabilité sans faute «renforce aussi l'application du principe pollueur payeur» (par. 80). Voir aussi Cie pétrolière impériale Ltée c. Québec (Ministre de l'Environnement), (2003) 2 R.C.S. 624, notamment le par. 24. Id., art. 7.

Id., art. 11.

Id., art. 15 .

Id., art. 17.

Id., art. 31 et 34.

L.R.Q., chapitre A-2.1.

Loi sur le développement durable, art. 19. Notons que la Cour d'appel du Québec, s'appuyant sur deux arrêts de la Cour suprême du Canada et sur le nouvel article 46.1 de la Charte affirme ce qui suit : «(...) la protection de l'environnement est devenue une valeur fondamentale de la société canadienne et un impératif collectif. Par ailleurs, le droit à un environnement sain a récemment été investi d'une valeur quasi constitutionnelle puisqu'il est désormais inscrit à l'article 46.1 de la Charte des droits et libertés de la personne». (voir Municipalité SaintLuc-de-Vincennes c. Compostage Mauricie Inc., jugement de la Cour d'appel du Québec rendu le 8 février 2008, par. 46)

14 Ces nouveaux concepts vont notamment se répercuter dans les appels d'offres.

15 L'Europe veut s'attaquer au «dumping environnemental» (les pays industrialisés qui ne respectent pas Kyoto seront pénalisés), Le Devoir, Montréal , 30 avril 2008; "L'Union européenne lance son plan d'action», (réchauffement climatique), Le Devoir, Montréal, 24 janvier 2008.

16 Commission on Growth and Development, The Growth Report (Strategies for Sustained Growth and Inclusive Development), The International Bank for Reconstruction and Development, The World Bank, Washington D.C., 2008; voir aussi «Vingt et un sages pour une mondialisation moins sauvage» (Un rapport d'experts remet en cause le fameux et très libéral «Consensus de Washington»), Le Devoir, Montréal, 26 mai 2008. La crise financière et économique mondiale, commencée à l'automne 2008, démontre l'importance du rôle de l'État. Du côté des Etats-Unis, plusieurs banques et producteurs de voitures (notamment General Motors et Chrysler) ont largement bénéficié de l'aide du Parlement des Etats-Unis. 